\title{
Sustained Responses in Chronic Hepatitis B Patients with Nucleos(t)ide Analogue Drug-resistance after Peg-interferon Alfa-2a Add-on Treatment: A Long-term Cohort Study
}

\author{
Yunhua Liu*, Weikun Li, Ting Jia, Dan Peng, Huimin Li, Xiaofei Li and Songqin LV \\ The Third People's Hospital of Kunming City, Kunming, China
}

\begin{abstract}
Background and Aims: The use of additional nucleos(t)ide analogues (NAs) without cross-resistance to previously used NAs as a rescue therapy is recommended by most international guidelines for chronic hepatitis B patients with NAresistance. We aimed to investigate the efficacy and safety of combination therapy of peg-interferon (PegIFN) alfa-2a and NA in these patients, comparing to those who switch to an alternative NA therapy without cross-resistance. Methods: In this prospective, comparative and cohort study, data were collected from the patients' hospital records. Eligible patients were those with hepatitis $B$ e antigen ( $\mathrm{HBeAg}$ ) positivity and resistance to one or more NAs. All patients were treated with alternative NA alone or in combination with PegIFN alfa-2a for 52 weeks or 72 weeks, respectively. HBeAg seroconversion was measured at the end of follow-up (EOF; more than 104 weeks after the end of treatment). Results: Sixty-three patients were recruited to the cohort study (NAtherapy group = 31 patients; combination therapy group of NA and PegIFN alfa-2a = 32 patients). At the EOF, significantly more patients in the combination therapy group (13/27, $48.2 \%$ ) achieved primary outcome of HBeAg seroconversion than those in the NA therapy group $(4 / 32,12.5 \%)(p=$ $0.003)$. Four patients $(14.8 \%)$ in the combination therapy group achieved hepatitis B surface antigen (HBsAg) loss and HBsAg seroconversion, but none in the NA therapy group did $(p=0.039)$. In the combination therapy group, 16 patients $(51.6 \%)$ achieved $\mathrm{HBeAg}$ seroconversion at the end of treatment, of which, 11 patients $(68.8 \%)$ maintained the response until EOF. Conclusions: Adding on PegIFN alfa-2a in combination with NA therapy might be an appropriate rescue treatment option for patients who have prior NA resistance. In addition, combination therapy induced sustained offtreatment biochemical responses in these patients.
\end{abstract}

Keywords: Chronic hepatitis B; Peginterferon alfa-2a; NA drug resistance; HBeAg seroconversion; Cohort study.

Abbreviations: $A D V$, adefovir dipivoxil; $A L T$, alanine aminotransferase; $C H B$ chronic hepatitis $B$; EOF, end of follow-up; EOT, end of treatment; ETV, entecavir; $\mathrm{HBeAb}$, hepatitis B e antibody; HBsAb, hepatitis B surface antibody; HBeAg, hepatitis $B$ e antigen; $H B s A g$, hepatitis $B$ surface antigen; LAM, lamivudine; NA, nucleos(t)ide analogue; NPV, negative predictive value; OR, odds ratio; PegIFN alfa-2a, peg-interferon alfa-2a; PPV, positive predictive value; ROC, receiver operating characteristic; ULN, upper limit of normal.

Received: 21 June 2017; Revised: 13 November 2017; Accepted: 29 November 2017

*Correspondence to: Yunhua Liu, Department of Liver Disease, The Third People's Hospital of Kunming City, Kunming 650041, China. Tel: +8613888349196, Fax: +86-871-63514717, E-mail: yunhua9989@126.com
Citation of this article: Liu Y, Li W, Jia T, Peng D, Li H, Li X, et al. Sustained responses in chronic hepatitis $B$ patients with nucleos (t)ide analogue drug-resistance after peg-interferon alfa-2a add-on treatment: A long-term cohort study. J Clin Transl Hepatol 2018;6(1):18-24. doi: 10.14218/JCTH.2017.00041.

\section{Introduction}

Chronic infection with hepatitis B virus (HBV) is the most common cause of liver cirrhosis and hepatocellular carcinoma (HCC) in Asia. Antiviral therapy is effective in inhibiting progression of the chronic hepatitis $\mathrm{B}(\mathrm{CHB})$ disease in many patients. Two classes of antiviral agents are available: nucleos(t)ide analogues (NAs) that inhibit viral polymerase activity and interfere with viral replication, and interferons (including conventional and pegylated forms) that have antiviral and immunomodulatory effects.

NAs are effective in most patients but suffering from drug resistance, resulting in viral breakthrough and subsequent biochemical responses, such as alanine aminotransferase (ALT) flare, acute exacerbations of liver disease, hepatic decompensation and even mortality. ${ }^{1}$ Previous studies have shown that resistance to lamivudine increases with treatment duration, with approximately $24 \%$ and $70 \%$ of the treated patients developing resistance by year 1 and year 5 , respectively. ${ }^{1,2}$ Resistance to entecavir, a third-generation NA with a high barrier to resistance, increased from $0 \%$ to $1.2 \%$ after 5-years treatment. ${ }^{1,3}$

The use of alternative NAs without cross-resistance to previously used NAs as a rescue therapy is recommended in most international guidelines. ${ }^{1,4,5}$ Its usage is optimal to achieve and maintain undetectable HBV DNA level. However, the response rates for these patients have been shown to be lower than those in the treatment-naive patients. ${ }^{6,7}$ So far, long-term effects, safety and tolerability of rescue therapy have remained unknown. Additionally, there is a potential risk for the emergence of multidrug-resistant strains. Thus, searching for alternative treatment strategies for patients with NA resistance is needed.

In contrast, the usage of pegylated interferon alfa-2a (PegIFN alfa-2a), either alone or in combination with NAs, has been shown to have high response rates of hepatitis $B$ e antigen ( $\mathrm{HBeAg}$ ) seroconversion and hepatitis B surface antigen (HBsAg) loss in the treatment-naive patients. ${ }^{8-10}$ The virological responses tend to be sustained in the majority of patients for at least up to 5 years. ${ }^{11}$ In a subsequent analysis of a previous study, the LAM-experienced patients 
responded equally well to PegIFN alfa-2a as the treatmentnaive patients. ${ }^{8}$ However, it is unclear whether PegIFN alfa$2 \mathrm{a}$ therapy in $\mathrm{CHB}$ patients with NA resistance is superior to NA rescue therapy. The mechanism of the action of IFN is quite different than that of NAs for HBeAg/HBsAg loss and seroconversion. Furthermore, previous studies suggested that IFN could help the degradation of covalently closed circular DNAs. ${ }^{12}$

In this study, we aimed to investigate the efficacy and safety of combination therapy of PegIFN alfa-2a and NA in NAresistant patients, comparing to those who switch to an alternative NA without cross-resistance to the previous one. Predictors of virological responses to PegIFN alfa-2a were also investigated.

\section{Methods}

\section{Study design}

In this prospective, cohort study, data were collected from the patients' health records at the Third People's Hospital of Kunming City, China. The first visit time of the included subjects was between March 20, 2008 and February 29, 2012. This study was completed on July 31, 2014.

The study protocol was approved by the Ethical Committee of the Third People's Hospital of Kunming City, China. The methods were carried out in accordance with the Declaration of Helsinki. Written informed consent was obtained from all participants.

\section{Inclusion and exclusion criteria}

All HBeAg-positive patients with NA resistance who received rescue therapy were enrolled in this cohort, regardless of the difference in the subsequent therapy (monotherapy or combination therapy). Resistance was confirmed by virological breakthrough (defined as HBV DNA $>2000 \mathrm{IU} / \mathrm{mL}$ from the nadir of initial response during therapy, confirmed 1 month after the nadir) and detection of NA mutations (one or multiple drug resistance mutations).

Patients who were co-infected with human immunodeficiency virus or hepatitis $A, C$ or $D$, or had a history or other evidence of a medical condition associated with chronic liver disease other than viral hepatitis (e.g., hemochromatosis, autoimmune hepatitis, metabolic liver disease, alcoholic liver disease) or decompensated liver disease (Child-Pugh score $\geq 6$ ) were excluded from this study.

\section{Treatment and outcome measures}

All patients were treated with a NA for $1-5$ years, and then they were treated with an alternative NA that was not cross-resistant to the one previously used, either alone or in combination with PegIFN alfa-2a (180 $\mu \mathrm{g} /$ week) for 52 weeks or 72 weeks. Although the design of the study was randomized, the treatments selected were based on each patient's actual conditions, their willingness, and economical reason, as the PegIFN was offered at the patients' own expense and some could not afford it. The observation period of this study ended on Jul 31st, 2014. The decision of treatment regimen and treatment duration was solely the responsibility of the clinician and his/her patients. Therefore, the final selection of the treatment regimen might not be completely non-random.

During the treatment and follow-up, patients attended outpatient clinic every 3 months for routine examinations and laboratory tests. Assessments were made at the baseline, weeks 12, 24, 52, 72, 104 and 156, and at the end of followup (EOF). The primary outcome was $\mathrm{HBeAg}$ seroconversion (defined as $\mathrm{HBeAg}$ loss and detection of anti-HBe antibody) at the EOF. Secondary outcomes were HBeAg loss, HBsAg loss, HBsAg seroconversion (defined as HBsAg loss and detection of the anti-HBs antibody), and HBV DNA suppression ( $<100 \mathrm{IU} / \mathrm{mL}$ ) at EOF. For patients who received combination therapy of NA and PegIFN alfa-2a, outcomes at the end of treatment (EOT) were measured.

\section{Laboratory measurements}

Laboratory tests were performed every 3 months, according to the local treatment guideline. HBV markers and HBV DNA level were measured at the laboratory of the Third People's Hospital of Kunming City. HBsAg level was quantified using the Roche Elecsys HBsAg assay II (Roche Diagnostics, Penzberg, Germany). HBV DNA level was determined by the qPCR assay (DAAN Gene, Guangzhou, China), with dynamic range of $10^{-2} \times 10^{9} \mathrm{IU} / \mathrm{mL}$. HBeAg, hepatitis B e antibody (HBeAb) and hepatitis $B$ surface antibody (HBsAb) were determined by the respective ARCHITECT Qualitative Assays (Abbott, Chicago, IL, USA). HBV genotype and mutation were determined using DNA amplification, reverse dot blot analysis and DNA sequencing (DaAn Gene, Guangzhou, China).

\section{Statistical analysis}

Continuous variables were shown as mean \pm standard deviation, whereas categorical variables were presented as number (percentage). Qualitative and quantitative differences between groups or subgroups were analyzed by the chi-square test or Fisher's exact test for categorical parameters and by the $t$-test for continuous parameters, as appropriate. Logarithmic transformation was performed in the case of skewed data.

Multiple logistic regression analyses were conducted to identify predictive value of various parameters in the study. The most frequently cited predictors of responses included baseline factors (treatment regimen, genotype, ALT level, HBsAg level, HBV DNA level) and on-treatment factors (HBsAg level, changes of HBsAg level from baseline, HBV DNA level, changes of HBV DNA level from baseline). Receiver operating characteristic (ROC) analyses were used to identify cutoffs associated with the achievement and maintenance of $\mathrm{HBeAg}$ seroconversion at the EOF. Sensitivity, specificity, positive predictive value (PPV) and negative predictive value (NPV) were assessed to identify optimal cutoffs.

Statistical analyses were conducted by SPSS version 18.0. A two-sided $p$-value of $<0.05$ was considered statistically significant.

\section{Results}

\section{Baseline characteristics and clinical features}

We first identified 3194 potentially eligible CHB patients, who first visited our hospital between March 20, 2008 and February 29, 2012. These patients were confirmed as NAresistant due to confirmed virological breakthrough and the detection of NA mutations. We excluded 3131 of the patients who were diagnosed as decompensated liver disease $(n=160)$, who did not have positive HBeAg $(n=383)$ or who were unwilling or unable to sign the informed consent form $(n=2588)$. 
Finally, 63 patients were included in the study cohort (NAtreated, 32 patients; combination therapy of NA and PegIFN alfa-2a, 31 patients). Seventeen and fourteen of the patients received combination therapy for 52 and 72 weeks, respectively. Four patients in the combination therapy group were excluded from the analysis at the EOF, because they continued the treatment with NA or withdrew the treatment 12 weeks posttreatment. In the NA-treated group, four patients were excluded from the analysis at EOF, because they continued treatment with NA and were lost to follow-up at 12 weeks posttreatment.
Liu Y. et al: PegIFN treated in NA-resistance CHB patients

The baseline demographic and clinical features of the study subjects are presented in detail in Table 1 . The mean duration of previous treatment of NA was shorter in patients with the combination therapy ( 2.5 years) than that with the NA therapy (3.5 years), as shown in Table 2. Patients with higher HBV DNA level and higher ALT level were treated with the combination therapy. According to the previous clinical experience and research reports, patients with higher HBV DNA may have poor response due to viral activity, while patients with higher ALT could have better response due to immune status.

Table 1. Baseline characteristics of patients enrolled in this study

\begin{tabular}{|c|c|c|c|}
\hline Baseline characteristic & $\begin{array}{l}\text { PegIFN alfa-2a and NA combination } \\
\text { therapy, } n=31\end{array}$ & NA therapy, $n=32$ & $p$ \\
\hline Male, $n(\%)$ & $25(80.6)$ & $20(62.5)$ & 0.094 \\
\hline Age in years & $32.1 \pm 7.4$ & $31.2 \pm 6.9$ & 0.602 \\
\hline Duration of previous treatment of NA in years & $2.5 \pm 1.1$ & $3.5 \pm 1.3$ & 0.023 \\
\hline Duration of follow-up in this cohort study in weeks & $201.8 \pm 75.2$ & $165.8 \pm 27.8$ & \\
\hline Duration of follow-up posttreatment in weeks & $159.9 \pm 61.1$ & & \\
\hline $\mathrm{HBeAg}, \mathrm{COI}$ & $540.1 \pm 663.8$ & $477.8 \pm 537.3$ & 0.778 \\
\hline $\mathrm{HBsAg}, \log _{10} \mathrm{IU} / \mathrm{mL}$ & $4.2 \pm 0.6$ & $4.1 \pm 0.4$ & 0.780 \\
\hline HBV DNA, $\log _{10} \mathrm{IU} / \mathrm{mL}$ & $6.1 \pm 1.1$ & $5.1 \pm 1.2$ & 0.001 \\
\hline ALT, $\times \mathrm{ULN}^{*}$ & $3.2 \pm 3.2$ & $1.5 \pm 1.3$ & 0.001 \\
\hline Genotype, $n$ & & & 0.448 \\
\hline B & 16 & 15 & \\
\hline $\mathrm{C}$ & 12 & 16 & \\
\hline Unknown & 3 & 1 & \\
\hline Resistance mutation & & & 0.108 \\
\hline 1 site & 23 & 15 & \\
\hline$\geq 2$ sites & 7 & 13 & \\
\hline Undetected & 1 & 4 & \\
\hline
\end{tabular}

* The upper limit of normal (ULN) of alanine aminotransferase (ALT) was $40 \mathrm{U} / \mathrm{L}$ in this analysis.

Table 2. NA treatment situation before recruitment

\begin{tabular}{lll}
\hline & PegIFN alfa-2a and NA combination therapy, $n=31$ & NA therapy, $n=32$ \\
\hline NA & $7(22.58 \%)$ & $1(3.13 \%)$ \\
ADV & $1(3.23 \%)$ & 0 \\
ADV+ETV & $2(6.45 \%)$ & $3(9.38 \%)$ \\
ADV+LAM & $5(16.13 \%)$ & $13(40.63 \%)$ \\
ADV+LDT & $1(3.23 \%)$ & $1(3.13 \%)$ \\
ETV & $1(3.23 \%)$ & 0 \\
ETV+PGE & $10(32.26 \%)$ & $4(12.50 \%)$ \\
LAM & $3(9.68 \%)$ & $9(28.13 \%)$ \\
LDT & $1(3.23 \%)$ & 0 \\
IFN & 0 & $1(3.13 \%)$ \\
IFN+ETV & $2.40 \pm 1.003$ & $2.52 \pm 1.298$ \\
Duration of treatment & $2.5(1.0,4.5)$ & $2.0(1.0,5.0)$ \\
Mean \pm standard deviation & & 0.7026 \\
Median (minimum, maximum) & \\
\hline
\end{tabular}


Of the patients in this analysis, the majority (59/63) were followed more than 156 weeks (combination therapy: 201.8 \pm 75.2 weeks; NA therapy: $165.8 \pm 27.8$ weeks). Most patients $(27 / 31,5$ patients lost during the NA treatment) who received the combination therapy were followed-up for more than 104 weeks after discontinuation of the combination therapy.

\section{Response rates at the EOF}

At the EOF, significantly more patients in the combination therapy group $(13 / 27,48.2 \%)$ achieved the primary outcome of $\mathrm{HBeAg}$ seroconversion than those in the NA therapy group $(4 / 32,12.5 \%)(p=0.003$; Table 3$)$. Four patients $(14.8 \%)$ in the combination therapy group achieved HBsAg loss and
HBsAg seroconversion, but none in the NA therapy group did $(p=0.039)$. More patients $(20 / 32,62.5 \%)$ in the NA therapy group $(15 / 27,55.6 \%)$ achieved HBV DNA suppression compared with that in the combination therapy group, but without statistical significance $(p=0.589)$. No patients with the combination therapy relapsed at the end of more than 104 weeks follow-up.

\section{HBsAg and HBV DNA decline by the treatment regimen}

The changes of HBsAg levels from baseline were significantly greater in the combination therapy group than that in the NA therapy group at every time point observed (Fig. 1A, C and D). Similar results were observed for the decline of HBV DNA levels (Fig. 1B).

Table 3. Response rates at the end of follow-up*

\begin{tabular}{|c|c|c|c|}
\hline$n(\%)$ & $\begin{array}{l}\text { PegIFN alfa-2a and NA } \\
\text { combination therapy, } n=27\end{array}$ & NA therapy, $n=32$ & $p$ \\
\hline HBeAg seroconversion & $13(48.15)$ & $4(12.50)$ & 0.003 \\
\hline HBeAg loss & $14(51.85)$ & $4(12.50)$ & 0.001 \\
\hline HBsAg seroconversion & $4(14.81)$ & 0 & 0.039 \\
\hline HBsAg loss & $4(14.81)$ & 0 & 0.039 \\
\hline HBV DNA suppression" & $15(55.56)$ & $20(62.50)$ & 0.589 \\
\hline
\end{tabular}

*End of follow-up: patients with PegIFN alfa-2a and NA combination therapy were treated for 52 weeks $(n=17)$ or 72 weeks $(n=14)$, and followed-up for at least 104 weeks. \#HBV DNA suppression was defined as HBV DNA $<100 \mathrm{IU} / \mathrm{mL}$.
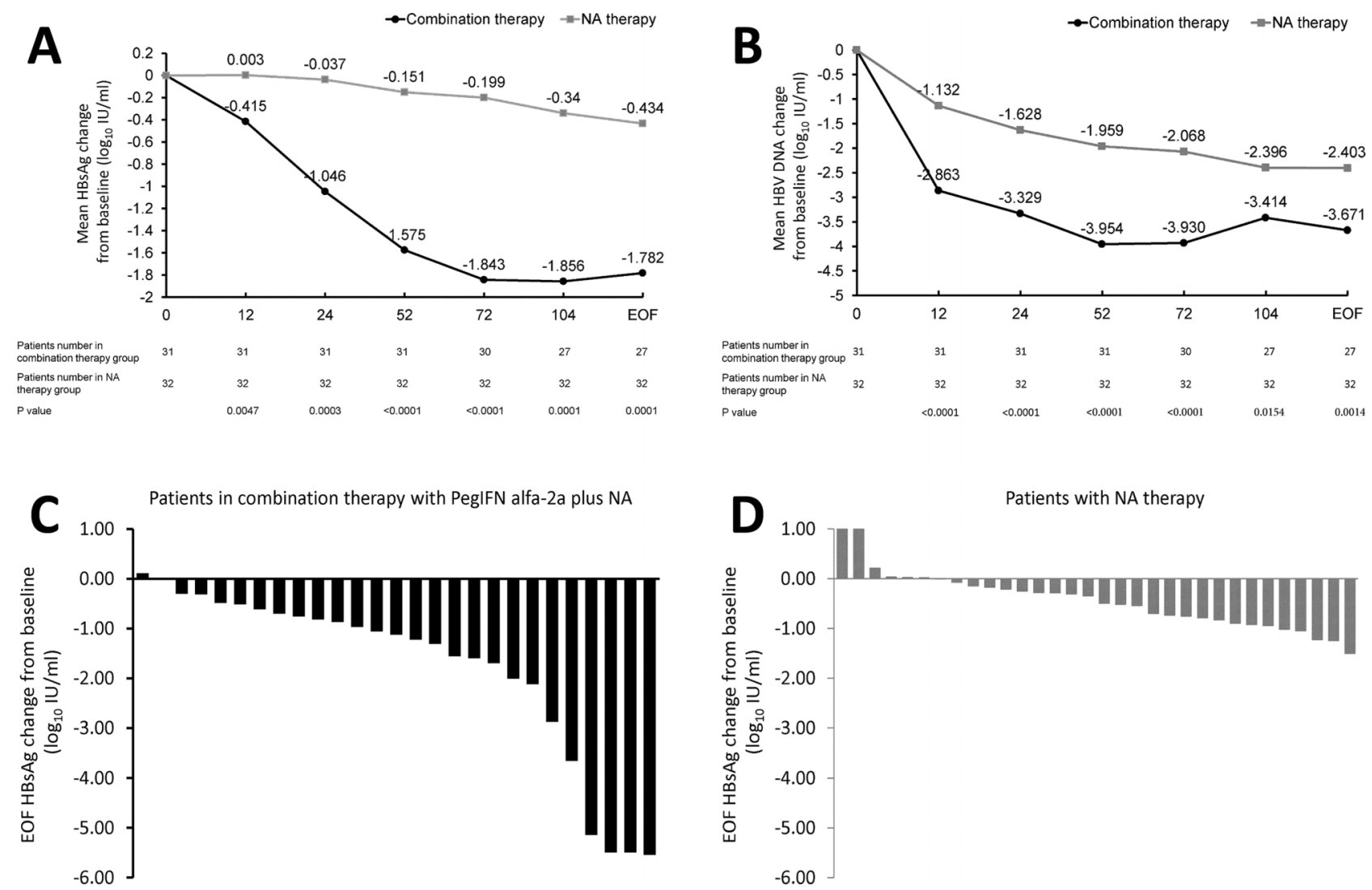

Fig. 1. Mean changes of HBsAg and HBV DNA. (A) Mean HBsAg change from baseline; (B) Mean HBV DNA change from baseline; (C) HBsAg change from baseline per patient in combination therapy with PegIFN alfa-2a plus NA at EOF; (D) HBsAg change from baseline per patient with NA therapy at EOF. 
Table 4. Sustained response rates in combination therapy group

\begin{tabular}{llll}
\hline$n(\%)$ & $\begin{array}{l}\text { EOT, } \\
n=31\end{array}$ & $\begin{array}{l}\text { EOF, } \\
n=27\end{array}$ & $\begin{array}{l}\text { Sustained } \\
\text { response }\end{array}$ \\
\hline $\begin{array}{l}\text { HBeAg } \\
\text { seroconversion }\end{array}$ & $16(51.62)$ & $13(48.15)$ & $\begin{array}{l}11 / 16 \\
(68.8)\end{array}$ \\
HBeAg loss & $17(54.84)$ & $14(48.15)$ & $\begin{array}{l}13 / 17 \\
(76.5)\end{array}$ \\
$\begin{array}{l}\text { HBsAg } \\
\text { seroconversion }\end{array}$ & $5(16.13)$ & $4(14.81)$ & $4 / 5(80.0)$ \\
$\begin{array}{l}\text { HBsAg loss } \\
\text { HBV DNA }\end{array}$ & $5(16.13)$ & $4(14.81)$ & $4 / 5(80.0)$ \\
suppression & $28(90.33)$ & $15(55.56)$ & $\begin{array}{l}15 / 28 \\
(53.6)\end{array}$ \\
\hline
\end{tabular}

*HBV DNA suppression was defined as HBV DNA $<100 \mathrm{IU} / \mathrm{mL}$.

\section{Sustained responses in patients with the combination therapy}

In the combination therapy group, 16 patients (51.6\%) achieved $\mathrm{HBeAg}$ seroconversion at the EOT. Of which, 11 patients $(68.8 \%)$ maintained the response until the EOF. The sustained response rates of secondary outcomes were higher than $75 \%$, except for HBV DNA suppression (Table 4). No correlation was found to exist between treatment duration and response rates in patients with the combination therapy

Slightly more patients $(8 / 14,57.1 \%)$ with 72 -weeks combination therapy achieved $\mathrm{HBeAg}$ seroconversion at the EOT compared to those with 52 -weeks of therapy $(8 / 17$, $47.1 \%)$, but the difference was not significant $(p=0.442)$. The slight advantage was not maintained until the EOF (72weeks: $5 / 11,45.5 \%$; 52-weeks: $7 / 16,43.8 \% ; p=0.619$ ). Similar trends in secondary outcomes (HBeAg loss, HBsAg seroconversion, HBsAg loss and HBV DNA suppression at the EOT) were observed.

\section{Predictors of treatment responses}

Treatment regimen and HBsAg level at baseline were identified as independent predictors for treatment response (odds ratio $(\mathrm{OR})=0.1786, p=0.009$ and $\mathrm{OR}=0.1311, p=0.007$, respectively). ROC analysis identified HBsAg level at week 12 as a good on-treatment predictor for $\mathrm{HBeAg}$ seroconversion at EOF (AUC: 0.8983). In ROC analyses, HBsAg level <4500 $\mathrm{IU} / \mathrm{mL}$ at week 12 of treatment was identified as the optimal cut off for prediction of $\mathrm{HBeAg}$ seroconversion at EOF (PPV = $76.5 \%$; NPV $=92.9 \%$ ).

Multiple logistic regression analyses were performed in patients with the combination therapy. The levels of HBsAg and HBV DNA were investigated as independent baseline predictors $(\mathrm{OR}=0.1789, p=0.044$ and $\mathrm{OR}=1.7380, p=$ 0.199 , respectively). ROC analysis identified HBsAg level at week 12 as a good on-treatment predictor for HBeAg seroconversion at $\mathrm{EOF}(\mathrm{OR}=0.1506, p=0.013$, AUC: 0.8722$)$. In ROC analysis, HBsAg level $<2000 \mathrm{IU} / \mathrm{mL}$ at week 12 of the treatment was identified as the optimal cutoff for prediction of $\mathrm{HBeAg}$ seroconversion at EOF (PPV $=75.0 \%$; NPV $=80.0 \%$ ).

The baseline ALT and HBV DNA were considered as independent variables in the model, as well as baseline HBsAg, NA type and treatment time before admission, and different treatment options after admission. However, only
Liu Y. et al: PegIFN treated in NA-resistance CHB patients

Table 5. Baseline ALT, HBV DNA and HBsAg impact on response

\begin{tabular}{lll}
\hline & OR & $p$ \\
\hline EOF HBeAg seroconversion & & \\
Baseline ALT & 1.0234 & 0.0028 \\
Baseline HBV DNA & 1.8106 & 0.0913 \\
Baseline HBsAg & 0.0242 & 0.0010 \\
EOF HBeAg loss & & \\
$\quad$ Baseline ALT & 1.0229 & 0.0022 \\
Baseline HBV DNA & 1.4744 & 0.2035 \\
Baseline HBsAg & 0.0718 & 0.0029 \\
EOF HBV DNA undetectable & & \\
Baseline ALT & 1.0152 & 0.1422 \\
Baseline HBV DNA & 1.1630 & 0.6097 \\
Baseline HBSAg & 0.2208 & 0.0600 \\
\hline
\end{tabular}

Abbreviations: ALT, alanine aminotransferase; EOF, end of follow-up; $\mathrm{HBSAg}$, hepatitis B surface antigen.

baseline HBsAg was identified as a significant indicator. So, the final model retained three factors, baseline ALT, HBV DNA and baseline HBsAg. The effect of baseline level on the outcome of the treatment can be dismissed, except for HBsAg, as shown in Table 5.

\section{Safety}

For the entire observation period, 24 patients (24/31, $77.4 \%)$ experienced at least one adverse event. In total, 9 patients (29\%) had platelet count decrease, 7 (22.6\%) had neutrophil count decrease, $6(19.4 \%)$ had weight loss, $6(19.4 \%)$ had asthenia, $4(12.9 \%)$ had thyroid dysfunction, $3(9.7 \%)$ had poor quality sleep and $2(6.5 \%)$ had rash. Most of the adverse events were mild to moderate. None of the patients developed serious adverse events. Four patients developed virological relapse (defined as HBV DNA $>2,000 \mathrm{IU} / \mathrm{mL}$ after stopping treatment for more than twice determined at more than 1 month apart). None of the patients developed ALT flare (defined as ALT > 2x ULN) nor clinical relapse (defined as viral relapse along with ALT $>2 x$ ULN after stopping therapy). No patient required dose modifications or discontinued treatment for safety reasons. Death or requirement of liver transplantation was not observed.

\section{Discussion}

In this study, we have shown that the combination therapy of PegIFN alfa-2a plus NA without cross-resistance achieved better long-term outcomes than the therapy with NA without cross-resistance alone in CHB patients with prior NA resistance. At EOF, the response rates measured by $\mathrm{HBeAg}$ and HBsAg seroconversion were significant higher in the combination therapy group than those in the NA therapy group. Both response rate and sustained serological response rate were higher in patients who received the combination therapy than in those who received the NA therapy.

China has a huge CHB population, of about 30 million. After nearly 10 years of treatment with NAs, most of these 
individuals desire to end the treatment, or they stop the treatment by themselves, resulting in the rise of drug resistance, even multiple drug resistance. Subsequent treatments were severely compromised for these patients. ${ }^{13}$ Optimal treatment (two NA combinations, or NA subsequently switching to another NA) cannot solve all the problems of drug resistance in these patients. ${ }^{14}$ Besides, the patients desire to have a limited duration, rather than taking medicine for a long time or for the whole life. Last but not the least, the patients should have the chance to achieve clinical cure by selected treatments according to the Chinese HBV guideline and Asian Pacific Association for the Study of the Liver HBV guideline. ${ }^{15}$

Patients in this cohort study were followed-up for more than 156 weeks, and even for 159.9 weeks posttreatment in the combination therapy group. The assessment of primary endpoint (HBeAg seroconversion at EOF) in patients treated with PegIFN alfa-2a plus NAs was made more than 3 years posttreatment, and high rates of both response $(48.15 \%)$ and sustained response $(68.8 \%)$ were observed. These data represented the long-term clinical outcomes. The viral suppression in both treatment groups was suboptimal. The response rates of HBV DNA suppression in the CHB patients with NA-resistance who received NA without cross-resistance with or without PegIFN alfa-2a (55.56\% and $60.5 \%$, respectively) were lower than that in the NA-naive patients. ${ }^{8}$

The imbalance of the baseline clinical characteristics was the limitation of our study. This is because that there were more patients with higher ALT and HBV DNA levels in the combination therapy group. Interestingly, compared with the NA therapy group, the HBV DNA decreased more significantly in the combination therapy group, suggesting that PegIFN not only has an immunomodulation role but also a role in inhibiting HBV DNAs. Although the baseline was not matched, the analysis of this study was based on the correction of all factors, which can effectively reduce the impact of mismatch.

Recently, the HBsAg levels at baseline and on-treatment were recognized as a potential predictor of the posttreatment response and were demonstrated as able to identify the optimal patients who had the highest likelihood of achieving $\mathrm{HBeAg}$ seroconversion. ${ }^{16}$ In our study, HBsAg level $<2000$ $\mathrm{IU} / \mathrm{mL}$ at week 12 of combination therapy with PegIFN alfa2a plus NA was a significant predictor of $\mathrm{HBeAg}$ seroconversion at EOF. Interestingly, HBsAg levels $<1500 \mathrm{IU} / \mathrm{mL}$ at week 12 of PegIFN treatment have been reported to be predictive of HBeAg loss with HBV DNA $<2000 \mathrm{IU} / \mathrm{mL}$ in treatment-naive $\mathrm{CHB}$ patients with $\mathrm{HBeAg}$ positivity receiving PegIFN alfa-2a therapy at 6 months post-treatment. ${ }^{17}$ Due to the previously mentioned limitation of this study, this cutoff remained to be validated. It was clear that low on-treatment HBsAg level was a useful predictor, consistent with previous studies. ${ }^{18,19}$ In addition, the 72-week treatment duration seemed to be more efficacious than the 52 -week treatment by virological response, $\mathrm{HBsAg}$ loss and $\mathrm{HBeAg}$ seroconversion (data not shown), but it was a trend observed without statistical significance.

The limitations of the study include some imbalances in the clinical characteristics between the groups (e.g., baseline characteristics, choice of treatment pattern, PegIFN treatment duration), which impact the final interpretation of the results. Another limitation of this study was that the number of patients enrolled was still small. Additional studies are warranted to confirm our findings.

In conclusion, adding on PegIFN alfa-2a to the NA therapy might be an appropriate rescue treatment option for patients who have prior NA resistance. Most importantly, such combination therapy can induce sustained off-treatment biochemical responses.

\section{Acknowledgments}

We thank all study participants, including patients, their families, the investigators and the nurses, who participated in this study. The study was supported by the Third People's Hospital of Kunming City, Kunming, China.

\section{Conflict of interest}

The authors have no conflict of interests related to this publication.

\section{Author contributions}

Conceived the topic, prepared the manuscript, generated the schematic diagrams, contributed to nutritional section, and edited the manuscript $(\mathrm{YL})$, contributed to the nutritional section ( $W L, T J, D P, H L, X L, S L)$.

\section{References}

[1] EASL clinical practice guidelines: Management of chronic hepatitis $B$ virus infection. J Hepatol 2012;57:167-185. doi: 10.1016/j.jhep.2012.02.010.

[2] Yao GB, Zhu M, Cui ZY, Wang BE, Yao JL, Zeng MD. A 7-year study of lamivudine therapy for hepatitis $B$ virus e antigen-positive chronic hepatitis $B$ patients in China. J Dig Dis 2009;10:131-137. doi: 10.1111/j.1751-2980. 2009.00375.x.

[3] Chang TT, Lai CL, Kew Yoon S, Lee SS, Coelho HS, Carrilho FJ, et al. Entecavir treatment for up to 5 years in patients with hepatitis $\mathrm{B}$ e antigen-positive chronic hepatitis B. Hepatology 2010;51:422-430. doi: 10.1002/hep.23327.

[4] Sarin SK, Kumar M, Lau GK, Abbas Z, Chan HL, Chen CJ, et al. Asian-Pacific clinical practice guidelines on the management of hepatitis B: a 2015 update. Hepatol Int 2016;10:1-98, doi: 10.1007/s12072-015-9675-4.

[5] Terrault NA, Bzowej NH, Chang KM, Hwang JP, Jonas MM, Murad MH. AASLD guidelines for treatment of chronic hepatitis B. Hepatology 2016;63: 261-283. doi: 10.1002/hep.28156.

[6] Biçer KC, Ari A, Genç VE, Özsu Caymaz S, Avci M, Bal F. Efficacy of entecavir treatment among chronic hepatitis $B$ nucleos(t)ide-naïve and -experienced patients. Turk J Med Sci 2015;45:99-104.

[7] Zoulim F, Locarnini S. Hepatitis B virus resistance to nucleos(t)ide analogues. Gastroenterology 2009;137:1593-1608.e1-2. doi: 10.1053/j.gastro.2009. 08.063.

[8] Lau GK, Piratvisuth T, Luo KX, Marcellin P, Thongsawat S, Cooksley G, et al. Peginterferon Alfa-2a, lamivudine, and the combination for HBeAg-positive chronic hepatitis B. N Engl J Med 2005;352:2682-2695. doi: 10. 1056/NEJMoa043470.

[9] Liaw YF, Jia JD, Chan HL, Han KH, Tanwandee T, Chuang WL, et al. Shorter durations and lower doses of peginterferon alfa-2a are associated with inferior hepatitis $B$ e antigen seroconversion rates in hepatitis $B$ virus genotypes B or C. Hepatology 2011;54:1591-1599. doi: 10.1002/hep.24555.

[10] Sonneveld MJ, Zoutendijk R, Hansen BE, Janssen HL. Pegylated interferon results in higher serological, but not virological, response rates when compared to continuous entecavir. Antivir Ther 2012;17:1605-1608. doi: 10. 3851/IMP2319.

[11] Marcellin P, Bonino F, Yurdaydin C, Hadziyannis S, Moucari R, Kapprell HP, et al. Hepatitis B surface antigen levels: association with 5 -year response to peginterferon alfa-2a in hepatitis $B$ e-antigen-negative patients. Hepatol Int 2013;7:88-97. doi: 10.1007/s12072-012-9343-X.

[12] Lucifora J, Xia Y, Reisinger F, Zhang K, Stadler D, Cheng X, et al. Specific and nonhepatotoxic degradation of nuclear hepatitis $B$ virus cccDNA. Science 2014;343:1221-1228. doi: 10.1126/science.1243462.

[13] Bai HL, Luo HT, Ye YN, Long H, Zhong RX. Observation on effect of peg interferon Iphaa-2a treating lamivudine resistant chronic hepatitis $\mathrm{B}$. Zhonghua Shi Yan He Lin Chuang Bing Du Xue Za Zhi 2010;24:230-231.

[14] Suh DJ, Lee HC, Byun KS, Cho M, Kweon YO, Tak WY, et al. Efficacy and safety of pegylated interferon- $\alpha 2 \mathrm{a}$ in patients with lamivudine-resistant $\mathrm{HBeAg}$ positive chronic hepatitis B. Antivir Ther 2013;18:765-773. doi: 10 . 3851/IMP2664. 


\section{Liu Y. et al: PegIFN treated in NA-resistance CHB patients}

[15] Sun J, Hou JL, Xie $\mathrm{Q}$ Li XH, Zhang JM, Wang YM, et al. Randomised clinical trial: efficacy of peginterferon alfa- $2 a$ in $\mathrm{HBeAg}$ positive chronic hepatitis $B$ patients with lamivudine resistance. Aliment Pharmacol Ther 2011;34:424431. doi: 10.1111/j.1365-2036.2011.04750.x.

[16] Yue W, Yuan H, Mao XR, Deng YD, Chen L. Clinical efficacy of various antiviral-based strategies to treat chronic hepatitis patients with positivity for hepatitis B e antigen and rtN236T mutation. Zhonghua Gan Zang Bing Za Zhi 2013;21:184-188.

[17] Sonneveld MJ, Hansen BE, Piratvisuth T, Jia JD, Zeuzem S, Gane E, et al. Response-guided peginterferon therapy in hepatitis $B$ e antigen-positive chronic hepatitis B using serum hepatitis B surface antigen levels. Hepatology 2013;58:872-880. doi: 10.1002/hep.26436.

[18] Ning Q, Han M, Sun Y, Jiang J, Tan D, Hou J, et al. Switching from entecavir to PegIFN alfa-2a in patients with HBeAg-positive chronic hepatitis $\mathrm{B}$ : a randomised open-label trial (OSST trial). J Hepatol 2014;61:777-784. doi: 10. 1016/j.jhep.2014.05.044

[19] Han M, Jiang J, Hou J, Tan D, Sun Y, Zhao M, et al. Sustained immune control in $\mathrm{HBeAg}$-positive patients who switched from entecavir therapy to pegylated interferon- $\alpha 2 \mathrm{a}$ : 1 year follow-up of the OSST study. Antivir Ther 2016;21:337-344. doi: 10.3851/IMP3019. 